\title{
BMJ Open Evaluation of the Gold Coast Integrated Care for patients with chronic disease or high risk of hospitalisation through a non-randomised controlled clinical trial: a pilot study protocol
}

\author{
Paul A Scuffham, ${ }^{1}$ Gabor Mihala, ${ }^{1}$ Lauren Ward, ${ }^{2}$ Anne McMurray, ${ }^{3}$ Martin Connor ${ }^{2}$
}

To cite: Scuffham PA, Mihala G, Ward L, et al. Evaluation of the Gold Coast Integrated Care for patients with chronic disease or high risk of hospitalisation through a non-randomised controlled clinical trial: a pilot study protocol. BMJ Open 2017;7:e016776. doi:10.1136/ bmjopen-2017-016776

- Prepublication history and additional material are available online. To view these files please visit the journal online (http://dx doi.org/10.1136/bmjopen-2017016776)

Received 10 March 2017 Revised 11 May 2017 Accepted 23 May 2017

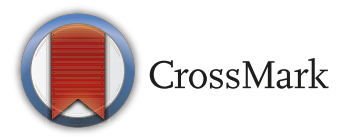

${ }^{1}$ Centre for Applied Health Economics, School of Medicine, Menzies Health Institute Queensland, Griffith University, Nathan, Australia

${ }^{2}$ Centre for Health Innovation, Griffith University and the Gold Coast Health and Hospital Service, Gold Coast, Australia ${ }^{3}$ Gold Coast Integrated Care, Gold Coast Hospital and Health Service, Gold Coast, Australia and the Menzies Health Institute Queensland, Griffith University, Gold Coast, Australia

Correspondence to Professor Paul A Scuffham; $p$. scuffham@griffith.edu.au

\section{ABSTRACT}

Introduction Chronic diseases are the leading cause of illness, disability and death in Australia. The prevalence and associated health expenditure are projected to soar. There is no 'whole system' approach to healthcare in Australia. To overcome this fragmentation, the Gold Coast Hospital and Health Service (GCHHS) is developing a new model known as Gold Coast Integrated Care (GCIC). To evaluate GCIC a 4-year pilot trial commenced in March 2015. This protocol paper describes the evaluation of GCIC.

Methods and analysis A pragmatic non-randomised controlled clinical trial is conducted to test the hypothesis that GCIC will result in improved health and well-being at no additional cost to the healthcare system. Using a mixed methods approach, impact, outcome and process evaluations will be undertaken to assess the effectiveness and acceptability, including the balance of costs between primary and public secondary care sectors, staff and training requirements, clinical service delivery, and trial implementation. Fifteen general practices have agreed to deliver GCIC. One thousand five hundred of their adult patients with treated chronic diseases, high risk of hospitalisation or healthcare utilisation were recruited to the intervention arm. Approximately 3000 patients not associated with the participating general practices were identified as controls using propensity matching which will provide service utilisation and disease data for usual care. Baseline data and follow-up observations are collected annually until the end of 2018. Quantitative analyses will measure patient healthcare costs, utilisation of health services, and health outcomes, and general practice clinical service delivery according to clinical guidelines (number of foot exams, HbA1c tests). Qualitative analyses will focus on patient and staff experiences, satisfaction, engagement and implementation of the programme as planned.

Ethics and dissemination Approval was received from the GCHHS and Griffith University. The study is registered with the Australian New Zealand Clinical Trial Registry (ACTRN12616000821493). Findings will be communicated via yearly reports to funding bodies and scientific publications.

Trial registration number ACTRN12616000821493; Preresults.

\section{INTRODUCTION}

Chronic diseases were the leading cause of illness, disability and death in Australia in 2011, ${ }^{2}$ and their relative burden on the health system increases over time. For example, health expenditure on the most prevalent chronic condition (type 2 diabetes) is projected to increase $520 \%$ from a $2002-$ 2003 level by 2032-2033, while the increase in total health expenditure is expected to be $189 \%$ over the same period, mainly due to two demographic growth factors: population ageing and the increase in population. ${ }^{3}$ The majority of chronic disease health dollars are allocated to hospital service for admitted patients, out-of-hospital services, medications and dental services. ${ }^{1}$ A major problem in managing chronic disease services is the fragmentation of the Australian healthcare system, attributed to the complex interplay of health funding and division of responsibilities between the federal, state and local governments for both private and public health services. Fragmentation is also pervasive between general practice and acute care, creating discontinuities in service provision. ${ }^{45}$

In Australia, there are numerous national and state initiatives and programmes aimed at linking sectors of the healthcare system; however, no consistent 'whole-system' approach to integrating services between primary healthcare and other healthcare services exists. ${ }^{6}$ A national agreement between all Australian federal, state and territory governments in 2012 supported an integrated approach to promote healthy lifestyles, prevention of illness and injury, and diagnosis and treatment across the continuum of care, as a means to improve health outcomes for all Australians and the sustainability of the Australian health system. ${ }^{7}$ 
These improvements are particularly relevant for the Gold Coast (Queensland, Australia) population, where almost a third of the population will be over 55 years of age, and the number of people aged over 85 years will nearly double by 2021 compared with the 2006 level. ${ }^{8}$ In the context of this national agreement and growing burden of disease, the Gold Coast Hospital and Health Service (GCHHS) and the Gold Coast Primary Health Network (GCPHN) and Queensland Health in partnership with Griffith University (GU), led the development of a new model of care. To evaluate this new model of service delivery a 4-year pilot trial, referred to as Gold Coast Integrated Care (GCIC), commenced in March 2015 with the establishment of a coordination centre to coordinate health services linking the patient and general practice with all other relevant health and hospital services. Significant funding was secured from Queensland Health and the GCHHS, with a contribution from the GCPHN. Additional funds were received from the Australian Government Department of Health to perform this evaluation study. None of the funding bodies had or will have an input in the design and management of the study, in the analysis and interpretation of data, or in the writing and submission of reports and publications. The GCHHS and the GCPHN are providing administrative data for analysis for the evaluation.

The design principles of GCIC are based on that of large-scale whole system models such as Kaiser Permanente $^{8}$ and Intermountain Healthcare ${ }^{9}$ in the USA. A review of these American models highlights the merits of integrated care programmes that focus on high-impact health conditions while situating primary care at the centre of chronic illness management, making it 'accessible, continuous, comprehensive, coordinated, and delivered in the context of family and the community'. ${ }^{810-13}$

Common attributes of successful integrated care programmes targeting individuals with chronic and complex conditions include the ability to stratify and target high-cost, high-need individuals, fostering effective interactions with patients providing self-management support and multidisciplinary care pathways organised through a single point of entry while creating an environment for successful leadership at all levels. ${ }^{12} 14$ The patient-centred medical home described by Jaén $e t$ al is an example of this type of approach which acts as a coordination centre for patients and their families, providing easy access to first-contact and comprehensive care where the patient is an active participant in their own health and well-being. ${ }^{15}$ A 2-year evaluation of this model in the USA showed improvements across both patient and health service outcomes with improved patient experience, quality, fewer emergency department and hospital visits, and lower costs. ${ }^{16}$

In the UK, health leaders, policy makers and researchers have a long established interest in integrated care with the decentralised capitated health service model rather than the fee for service framework in Australia. Lessons from UK programmes including the Integrated Care
Pilots and Trafford highlight the importance of strong leadership and collective governance with co-location of multidisciplinary teams within an integrated care framework. ${ }^{17-21}$ Additionally, researchers emphasise the need for communication, exploiting linked data sets including general practice data, and shared information technology and health record systems. ${ }^{1822}$

The GCIC programme was founded on the notion that care coordination, planning and patient advocacy is best achieved in collaboration with general practitioners (GPs), supported by specialists, multidisciplinary teams, non-government organisations and private allied health providers, so that patients get the care they need, when they need it, in ways that are user-friendly, achieve the desired results and provide value for money. ${ }^{23}$ The overarching goal of GCIC is to proactively manage patients with chronic and complex conditions, in close collaboration with GPs, to reduce presentations to emergency departments, improve the capacity of specialist hospital outpatient departments, and decrease planned and unplanned hospital admission rates, all of which should be cost-effective for the GCHHS. This protocol paper describes the evaluation of the GCIC programme, a 4-year pilot programme, guided by the Standard Protocol Items: Recommendations for Interventional Trials recommendations. ${ }^{24}$

\section{METHODS AND ANALYSIS}

\section{Study design}

The evaluation study is a pragmatic non-randomised controlled clinical trial to test the primary hypothesis that the GCIC will result in improved health and well-being at no additional cost to the healthcare system. The primary unit of analysis will be the individual, while the general practices and healthcare workforce will be the secondary units of analysis.

Using a mixed methods approach, impact, outcome and process evaluations will be undertaken to assess the overall effectiveness and acceptability of GCIC. The evaluation includes two components: a core evaluation of high-risk patients and a population health outcomes component. The following research questions were defined. Co-primary questions: (A) Did GCIC reduce overall costs of delivering healthcare services to the GCHHS for high-risk patients with complex needs compared with usual care? (B) Did GCIC improve health outcomes for high-risk patients with complex needs compared with usual care? Outcome evaluations: (A) Did GCIC change the proportion of costs shared by the primary and secondary care sectors? (B) Did GCIC reduce potentially avoidable hospital admissions, emergency presentations and length of stay? (C) To what extent did GCIC improve experiences and satisfaction with care for both patients and clinicians? (D) What was the relationship between patient outcomes and clinical and demographic characteristics? (E) What was the cost-effectiveness of GCIC? Impact evaluation: (A) What are the costs and benefits of generalising the GCIC model 
to other parts of Australia? (B) What are the projected changes in numbers of hospital admissions, emergency presentations, general practice visits and other healthcare utilisation? (C) What is the staffing requirement (including training needs) and displacement from generalising GCIC? Process evaluation: (A) Did GCIC improve clinical service delivery according to guidelines? (B) To what extent was GCIC implemented as intended? (C) Which elements of GCIC were seen to be most useful by staff and patients, respectively? (D) To what extent did GCIC improve continuity of care?

Governance arrangements for GCIC include a managing director and a senior management team referred to as the Executive Management Team, which provides strategic leadership and management of the overall processes and business operations as well as strategy, budget, programme structure and administration. A Strategic and Clinical Advisory Committee has been appointed for providing clinical oversight and strategic direction. An Evaluation Steering Committee acts as the peak advisory body for the evaluation study, providing oversight and advice to the team to ensure the continued quality and credibility of evaluation activities including facilitating access to administrative data and ensuring the evaluation is on track. Individuals responsible for the design and implementation of GCIC are employees of GCHHS and other organisations (excluding GU). GU provides an independent team based at the School of Medicine to perform trial data management, analyses, interpretation and reporting.

\section{Participants and recruitment}

An expression of interest was sent to all general practices on the Gold Coast $(\mathrm{n}=165)$ to invite them to participate in GCIC. General practices that indicated an interest received a visit from representatives of the programme and the GCPHN. As a result, 15 general practices have signed on to deliver the proposed integrated model of care as part of GCIC (referred to as network general practices). The GCIC programme has also engaged the 23 Gold Coast general practices that had a data sharing arrangement with PHN, and were available to act as practice controls. These 'non-network' practices were approached in person, and invited to provide written consent to be involved in the study. Their involvement in GCIC is limited to providing aggregate (deidentified) service utilisation and clinical metrics data, which will be used to compare population health outcomes with the network practices. The larger sample size of non-network practices is an attempt to overcome the potential bias due to systematic differences between practices, including PHN chronic disease interventions.

The network general practices have a total active (ie, attended the practice three or more times in the past 2 years $^{25}$ ) population of approximately 92000 patients (about 17\% of the Gold Coast population). Literature indicates that approximately $3 \%$ to $5 \%$ of the general practice population are complex high-risk patients having multiple chronic conditions with the highest risk of hospitalisation ( $\mathrm{RoH})$, and $10 \%$ to $15 \%$ are 'diagnosed but stable' with a known chronic condition and at medium RoH. ${ }^{26}{ }^{27}$ Eligibility for the programme included GCHHS patients at high RoH identified through the following six processes: (A) a manual review of hospital and general practice records to identify patients who in the past 3 years had at least one inpatient admission, at least one emergency department presentation, at least 20 general practitioner visits, currently use 5 or more prescibed medications, and have a coded diagnosis of diabetes, chronic heart disease, chronic obstructive pulmonary disease or chronic kidney disease, ${ }^{28}$ (B) purposely designed $\mathrm{RoH}$ Score within the next 12 months based on 58 predictor variables covering medical history, demographics and prior healthcare utilisation from both general practice and hospital data. Patients with a RoH Score of $70 \%$ and higher were identified and their details sent to the GP to consider for enrolment to the programme, $(\mathrm{C})$ disease registers using $\mathrm{RoH}$ Score plus clinical metrics beyond normal range, (D) medical registrar reviews of patients' records when admitted to hospital from network practices, (E) GP referrals for patients who were not captured in the manual risk stratification process, (F) direct referral by family members of patients requesting to be part of the programme and who were assessed as amenable. For evaluation purposes eligibility was restricted to the adult ( $\geq 18$ years of age) high-risk population at the time of enrolment. Exclusion criteria include those with non-chronic conditions, maternity patients, residents of aged care facilities, residents of areas other than the Gold Coast, children $<18$ years at the time of recruitment. Approximately 1500 patients were recruited to form the intervention arm of GCIC between March 2015 and September 2016. Participants gave written informed consent to participate in GCIC, and separate consents to access their hospital, Medicare and pharmaceutical (Medicare Benefits Schedule (MBS)/ Pharmaceutical Benefit Scheme (PBS)) claims records (see online supplementary files A,B and C). Patients within the network general practices who have been diagnosed with at least one chronic condition and do not meet the high-risk criteria are categorised as 'diagnosed but stable', and are proactively managed through 'live' general practice based disease registers. These patients may transfer into the high-risk category and thus be eligible for holistic assessment, depending on the status of their condition.

Approximately 3000 patients with similar characteristics at baseline to patients in the intervention group have been allocated to a matched control group (1:2 = intervention:control) through a two-step process: initial identification and propensity score matching. The aim was to achieve the best possible match, however, restriction to patient level hospital data have limited the evaluation team's ability to match on all criteria used for identifying the intervention group. Initial identification of potential control group members was completed according to the following hospital criteria: 
- Diagnosis of at least one International Classification of Diseases (ICD)-10 block ( $\mathrm{n}=108)$ marked as primary or secondary reason for admission

- Any occasion of service at GCHHS between 01 July 2012 and 30 June 2015

- Aged $\geq 18$ years

- Resident of the Gold Coast region

- Not a patient of network general practices

- Not requiring an interpreter

- Not a resident of an aged care or nursing facility

- Alive in June 2015

Following the initial identification, the research team identified and selected control group participants through propensity matching, for inclusion in the evaluation study.

Propensity scores were calculated using a probit model, where the covariates included age, gender, number of outpatient appointments, number of emergency presentations, number of hospitalisations, length of stay at emergency, length of stay in hospital, and a number of binary hospitalisation history variables (to indicate where the primary reason of admission was one of 108 predetermined ICD-10 blocks of interest). Matching on propensity scores was completed using the 1:1 nearest neighbour matching without a replacement method.

Participants of the control group have been contacted with an invitation to join the subgroup referred to as the active control group. The size of the active control group is approximately $20 \%$ of the size of the control group, but allowing for some deaths and losses (by oversampling by 25\%) recruitment into the active control group reached $n=750$. These participants have provided informed written consent to allow access to their MBS/ PBS claims records, and will complete follow-up surveys annually. Patients who did not consent to participate as an active control have been allocated to a passive control group with the purpose of tracking hospital utilisation data only. Figure 1 presents the total recruited cohort numbers. Public Health Act approval (RD005624) was received from the Queensland Government Department

\section{$N=4500$}

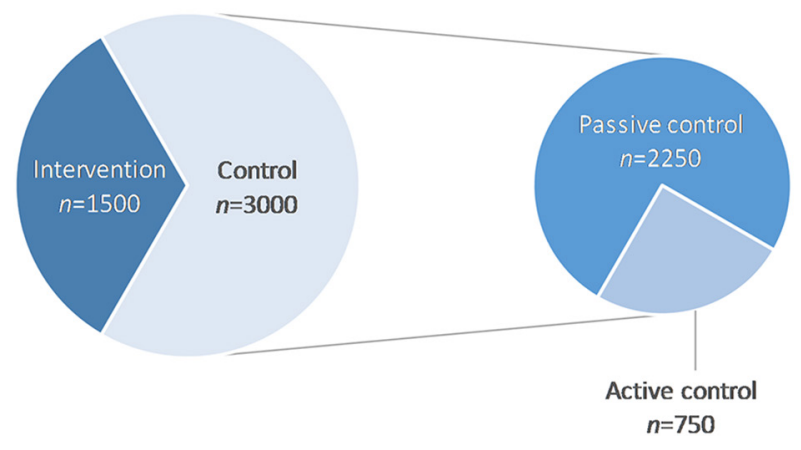

Figure 1 Study group sizes (protocol). of Health for access to confidential health information to undertake the matching process and data analysis.

\section{Intervention}

A key element of the GCIC programme is the proactive management of participating patients. Participating patients undertook a comprehensive holistic assessment which included a review of previous medical information, identification of current service providers, and health assessments to develop a detailed summary of their social needs for building a jointly agreed and flexible shared care plan. The holistic assessment incorporates a health profile which determines the need for further medical, nursing, pharmacy and allied health assessments to identify relevant clinical metrics for ongoing monitoring and exacerbation management. The care delivery team is centred on the GP as the primary care provider with assistance provided from both clinical and non-clinical staff depending on the patients' requirements and care plan. The care plan is developed collaboratively by the GP and members of the multidisciplinary team at the GCIC coordination centre. A shared care record accessible by the patient and members of their nominated healthcare team is central to facilitating timely communication of care needs between multiple healthcare providers and to accommodate patients' needs and preferences for care.

Major features of GCIC include: (A) participant identification through risk stratification, (B) joint clinical governance between GCHHS, primary care practitioners, and the social and community services sector to develop individual, flexible shared care agreements and plans, (C) proactive care managed through general practice patient registers, to ensure all people requiring care receive it, not just those who seek it, (D) care aimed at assessing and treating the whole patient, not just one condition, through the operation of integrated care clinics staffed by multidisciplinary health professionals, (E) a single contact phone number for general practice staff, patients, families and carers (ie, the coordination centre), (F) rapid access to additional home services, specialist teams within GCHHS or other participating clinics, $(G)$ enhanced information and communication systems between all services including shared electronic patient records to allow the care team to assist in the timely coordination of care, $(\mathrm{H})$ care supported by protocols, clinical guidelines, care pathways, discharge and referral guidelines, (I) shared decision making between patient and healthcare team with family and carer involvement as required, (J) register of patients maintained and accessible to the Medical Assessment Units at GCHHS, (K) direct admission to the Medical Assessment Units or inpatient wards for selected complex patients.

\section{Study data}

Data for the evaluation are being collected from a number of sources, including general practices, GCHHS, Medicare, surveys and focus groups. Baseline data were collected at recruitment, and follow-up observations 
Box 1 Data collection plan

Core evaluation of high-risk patients

- characteristics (age, sex, home postcode, health insurance status) at baseline $(A, B, C)$

- additional characteristics (education, income, employment, living arrangement, smoking, etc) at baseline and 12 monthly follow-ups (A,B)

- surveys (quality of life using Assessment of Quality of Life - 4 Dimensions (AQoL-4D), ${ }^{29}$ capability using ICEpop CAPability measure for Older people (ICECAP-0), ${ }^{30}$ social support using Lubben Social Network Scale (LSNS), ${ }^{31}$ assessment of care using Patient Assessment of Care for Chronic Conditions - 20 items (PACIC-20), ${ }^{32}$ satisfaction using Short Assessment of Patient Satisfaction (SAPS) ${ }^{33}$ ) at baseline and at 12 monthly follow-up intervals $(A, B)$

qualitative data (service acceptability, etc) at 12-month intervals (intervention patients), at 24 months (control patients), at 6 months, 18 months and completion (intervention staff) (D)

- qualitative data (implementation, acceptability, etc) at baseline and 12-month intervals (E)

- hospital inpatient details (medical classifications, length of stay, cost) over 3 years prior to enrolment and 6 monthly follow-ups (A,B,C)

- emergency presentations (priority, diagnoses, length of stay, cost, etc) over 3 years prior to enrolment and 6 monthly follow-ups (A,B,C)

hospital outpatient visits (specialty, cost, etc) over 3 years prior to enrolment and 6 monthly follow-ups $(A, B, C)$

hospital investigations (test type, cost, etc) over 3 years prior to enrolment and 6 monthly follow-ups (A,B,C)

medications prescribed (type, class, cost, etc) over 3 years prior to enrolment and 6 monthly follow-ups $(A, B, C)$

general practice visits (number, Medicare item numbers) (A)

tests, for example, weight, $\mathrm{HbA}_{1 \mathrm{c}}$, blood pressure, total cholesterol, and so on (result and date) (A)

- Medicare claim details (item numbers, date, cost, etc) over 1 year prior to enrolment and 12 monthly follow-ups $(A, B)$

- PBS claim details (item numbers, date, cost, etc) over 1 year prior to enrolment and 12 monthly follow-ups $(A, B)$

- mortality at 12 monthly follow-ups $(\mathrm{A}, \mathrm{B}, \mathrm{C})$

staff cost at 12 monthly follow-ups

- population projections (age, sex, region, size, healthcare utilisation, staffing, etc) for a time period of 2015-2018

Evaluation of population outcomes

- diabetes care and prevalence details ( $\mathrm{HbA}_{1 \mathrm{c}}$, foot, eye, blood pressure, lipid examinations, vaccinations, etc) at baseline and 3 monthly follow-ups ( $\mathrm{F}$ )

- chronic obstructive pulmonary disease care details (spirometry, vaccinations, etc) at baseline and 3 monthly follow-ups (F)

- chronic kidney disease care details (eGFR, blood pressure, lipid examinations, vaccinations, medications, adherence to guidelines, etc) at baseline and 3 monthly follow-ups (F)

heart disease care details (blood pressure, lipid examinations, vaccinations, medications, adherence to guidelines, etc) at baseline and 3 monthly follow-ups (F)

survey of chronic illness care provision at baseline and at trial completion $(\mathrm{G})$

Trial evaluation at completion

- risk stratification, holistic assessment, services accessed, patient records and disease registries, governance and organisational arrangements, training and skills, and so on

$\mathrm{A}=$ intervention group; $\mathrm{B}=$ active control group; $\mathrm{C}=$ passive control group; $\mathrm{D}=$ focus group; $\mathrm{E}=$ general practice staff surveys; $\mathrm{F}=$ patients of all network and non-network general practices; $\mathrm{G}=$ network general practices; $\mathrm{HbA}_{1 \mathrm{c}}=$ glycated haemoglobin; PBS = Pharmaceutical Benefit Scheme; eGFR = estimated glomerular filtration rate; Instrument reliability: internal consistency of $A Q D L-4 D$ is (Cronbach's) $\bigotimes=0.81,{ }^{34}$ LSNS- $6 \rrbracket=0.83^{31}$ and SAPS $\unrhd=0.86,{ }^{33}$ ICECAP-0 is not fully validated, ${ }^{35} 36$ test-retest reliability of PACIC-20 is $r=0.58 .^{37}$

are being collected at every 3-12 months until the end of 2018 (see box 1 and online supplementary file D). An incentive (gift cards) was introduced to mitigate the potential risk of low response rates from active control patients. Discontinuations are anticipated to be due to losses to follow-up (eg, admission into a residential aged care facility, or moving out of area) and deaths. Data on deaths are obtained from GCHHS and the Queensland Government death register. Administrative data on losses to follow-up are collected through GCHHS and GCPHN for discontinuations accessing local healthcare services. Identifiable participant information used for evaluation is managed separately from deidentified observations, and stored in locked filing cabinets or password protected in GU's secure research data storage. A research review committee (MC, AMcM, PAS) has ultimate authority on access to the data and agreements. Any complaints or spontaneously reported adverse events are reported to the primary contacts for the evaluation (PAS, LW) and to the ethics committee.

\section{Power, detectable difference and sample size}

The detectable difference in the total healthcare cost per patient was calculated based on: (A) assuming 15 general practices (clusters) per study group, (B) the number of participants enrolled at each of the clusters is reasonably balanced with an average of approximately 100, (C) mean costs for hospitalisations per participant (in the control group) over 2years of \$A10000 (Australian dollars in 2015; SD: $\$ A 4000),{ }^{38}$ (D) a $6 \%$ reduction in hospital admissions, ${ }^{39}$ (E) a coefficient of variance within each cluster of 0.47 , (F) an intracluster correlation of 0.01 , resulting in a difference of $\$ 630$ at the 0.05 significance level which can be detected with $80 \%$ power. Given the 1:2 ratio, smaller differences could be detected.

A second detectable difference calculation was undertaken at the participant level, assuming 78\% hospitalisation rate per year in the control group and $20 \%$ of participants lost to follow-up in both groups: at the level of $90 \%$ power and 0.05 significance there will be adequate 
sample size to detect a $5 \%$ reduction in hospitalisation rates between the study groups. The Group Health Cooperative reported a $6 \%$ difference in hospitalisations from their Integrated Care model. ${ }^{39}$

For the analysis of health outcomes and patient satisfaction, 215 control participants are sufficient to identify a mean difference in quality of life (measured using the AQoL-4D scored with utility weights from an Australian population on a scale of 0 to $1^{29}$ ) of 0.05 compared with intervention arm participants with $80 \%$ power at the 0.05 level of significance. This calculation was based on a SD of 0.20 for the intervention arm participants and 0.25 for the active control group participants. This active control group sample size allowed for factors such as $55 \%$ attrition.

\section{Quantitative analyses}

An economic evaluation of GCIC will be undertaken from the perspective of the Queensland and Australian governments (ie, the healthcare funders). This will present the additional cost per quality-adjusted life year gained. In addition, separate analyses will be undertaken around costs to GCHHS and the Commonwealth Government to identify additional costs and cost savings in the different sectors. Generalised linear models will be developed to allow us to model clinical and economic outcome factors, with dependent variables that follow a distribution that is Poisson (eg, number of emergency department visits), exponential (eg, length of hospital stay), normal or binomial. The functional form chosen for the analysis will be driven by the distributions of the data. Data will be analysed taking into account the time-series nature of the data. A series of regressions will be undertaken, with dependent variables of volume of services used, mortality, quality-adjusted life years, total costs to the health system and net health benefits. Where the dependent variable contains zeros, alternative forms of generalised linear models will be used such as Poisson, negative-binomial or zero-inflated regression approaches. Diagnostics of regression models will be examined, for example, residuals, influential values, and so on. The incremental cost per quality-adjusted life year gained (incremental cost-effectiveness ratio) will be calculated. Forward estimates (up to 5 years following the end of GCIC) will be undertaken to identify the likely costs and cost offsets from generalising GCIC. The budget impact will be presented as annual budget costs for up to 5 years for the GCHHS and primary care sectors, for the Gold Coast, Queensland and Australian populations. Deterministic sensitivity analyses will be undertaken around key parameters with the greatest uncertainty.

\section{Qualitative analyses}

Qualitative evaluation data will be collected and analysed around the following topics: (A) patient experiences of care, (B) level of satisfaction with GCIC, (C) influences on continuity of care throughout the patient journey, (D) overall staff experience and level of satisfaction, (E) staff member engagement in change management, $(\mathrm{F})$ strategy implementation, (G) most useful elements in achieving optimal patient outcomes, $(\mathrm{H})$ modifications to GCIC to achieve process improvements to meet goals, (I) team culture influencing outcomes, and (J) change management. Data will be collected via focus groups and surveys: (A) intervention patient focus groups: four, 60-min groups of 10-12 randomly selected patients every 12 months, to gauge satisfaction and discuss recommendations, open ended questions, discussions of experiences and perceptions of GCIC, (B) control patient focus groups: four, 60-min groups of 10-12 randomly selected patients from the active control group held at 24 months to examine experiences of 'usual care',(C) incremental 60-min staff focus groups held at 6 months, 18 months and completion, to gauge satisfaction and discuss recommendations, with all GCIC staff, (D) general practice staff surveys at baseline and 12-month intervals, (E) ongoing staff feedback through confidential online surveys, with monthly feedback reports, (F) historical document analysis (to track programme development), and (G) stakeholder feedback (through membership on Strategic and Clinical Advisory Committee). The focus group sessions will be recorded, transcribed and interpreted using the Braun and Clark ${ }^{40}$ method of content analysis. Qualitative data will be categorised for comparison with the quantitative findings to identify areas of congruence or issues to be addressed in the evaluation.

\section{Strengths and limitations}

While a strength of GCIC is the substantial number of participating patients, indicating that the evaluation will yield meaningful information to inform future service planning, GCIC is limited by the fact that it is currently a 3-year 'proof of concept' endeavour in one geographical location, and its expansion to other local health and hospital services will depend on the results of the economic evaluation. The lack of randomisation in patient recruitment to the programme may present a potential selection bias. Additionally, there may be selection bias from (A) general practices who responded to the letter of invitation to participate, with insufficient feedback to ascertain the reasons for non-participation, and (B) from the active control group who actively opt in. A potential confounding factor may be an inability to detect significant differences between groups due to competing interventions occurring in the control practices. Quarterly reports from PHN will provide details of programmes/interventions implemented in each practice to identify any contextual elements affecting the findings. Limitations in terms of patient choice should also be considered as all patients have a choice about where to seek healthcare as well as the fact that a chronic disease health population such as that enrolled in GCIC is closer to death than another population. Studies in the $\mathrm{UK}^{41-43}$ and evaluation of the chronic disease management plans in Australia ${ }^{5}$ have also reported a potential confounding factor because of regression to the mean. This occurs 
because those with high RoH have shown natural reductions in hospital use over time, with subsequent rates of hospitalisation being statistically less likely to be as high, even in the absence of intervention. We are attempting to overcome this situation by using propensity matching with a retrospective valid control group from routinely collected, computerised, patient level health and health services data. ${ }^{41}{ }^{43}$ Another potential confounding factor cautions us against drawing conclusions about patient outcomes linked exclusively to the model of care rather than the broader health system. ${ }^{44}$ Further, as reported in previous evaluations ${ }^{45}$ the general practices who volunteered to participate may have had both the will and resources for quality improvement so our controls have been selected from non-participating practices. Finally, duration of follow-up may be a study limitation, however the 3-4-year follow-up period is more than most clinical trials, and should give a good indication of the longerterm effectiveness of GCIC.

\section{ETHICS AND DISSEMINATION}

Ethics approval from Gold Coast Hospital and Health Service Human Research Ethics Committee (HREC) was received on 16 March 2015 and from Griffith University HREC on 16 April 2015. Written consent was required from all intervention group and active control group participants. This includes consent to be in the trial, consent for access to GP and hospital records, and additional consent to access Medicare records. The study is registered with the Australian New Zealand Clinical Trial Registry (registration number: ACTRN12616000821493) as a non-randomised controlled intervention study. Amendments to the protocol will be passed by the HREC and noted in resulting publications.

The results will be disseminated via yearly interim reports including a final report to the Commonwealth Department of Health and GCHHS board and executive. Summary reports will be disseminated to the wider GCHHS staff, GU team members, the PHN, the general practices and participating patients. It is expected that there will be several publications and conference presentations from this study. We anticipate that the evaluation findings will augment the evidence pertaining to the value of a whole-system integrated model of care in Australia.

Contributors PAS, AMcM, MC conceived of the study. PAS, GM, LW, AMcM, MC participated in design and coordination, and in the preparation of the study protocol. All authors read and approved the final manuscript.

Funding This research is supported by funding from Gold Coast Hospital and Health Service, Queensland Health, the Gold Coast Primary Health Network, the Australian Government Department of Health, and Griffith University.

\section{Competing interests None declared.}

Patient consent Detail has been removed from this case description/these case descriptions to ensure anonymity. The editors and reviewers have seen the detailed information available and are satisfied that the information backs up the case the authors are making.

Ethics approval Gold Coast Hospital and Health Service, and Griffith University.

Provenance and peer review Not commissioned; externally peer reviewed.
Data sharing statement Unpublished data from the study will be communicated via yearly reports to funding bodies and stakeholders (Commonwealth Department of Health, Queensland Health, Gold Coast Primary Health Network, Gold Coast Hospital and Health Service).

Open Access This is an Open Access article distributed in accordance with the Creative Commons Attribution Non Commercial (CC BY-NC 4.0) license, which permits others to distribute, remix, adapt, build upon this work non-commercially, and license their derivative works on different terms, provided the original work is properly cited and the use is non-commercial. See: http://creativecommons.org/ licenses/by-nc/4.0/

(c) Article author(s) (or their employer(s) unless otherwise stated in the text of the article) 2017. All rights reserved. No commercial use is permitted unless otherwise expressly granted.

\section{REFERENCES}

1. Australia's health 2014. Canberra: Australian Institute of Health and Welfare, 2014.

2. Cottrill A. The need for an integrated approach to chronic disease management in Australia. Health Voices 2015;17:28-9.

3. Goss J. Projection of Australian health care expenditure by disease, 2003 to 2033. Canberra: Australian Institute of Health and Welfare, 2008.

4. OECD reviews of Health Care Quality: Australia 2015. Paris: OECD Publishing, 2015.

5. Billot L, Corcoran K, McDonald A, et al. Impact Evaluation of a System-Wide Chronic Disease Management Program on Health Service Utilisation: A Propensity-Matched Cohort Study. PLoS Med 2016;13:e1002035.

6. Bywood PT, Jackson-Bowers E, Muecke S. Initiatives to integrate primary and acute health care, including ambulatory care services. Adelaide: Primary Health Care Research \& Information Service, 2011.

7. National Healthcare Agreement 2012. Canberra: Council of Australian Governments, 2012.

8. McCarthy D, Mueller K, Wrenn J, et al. Kaiser Permanente: bridging the Quality divide with Integrated Practice, Group Accountability, and Health Information Technology. New York: The Commonwealth Fund, 2009.

9. Silow-Carroll S, Edwards JN. Intermountain Healthcare's McKay-Dee Hospital Center: Driving Down Readmissions by Caring for Patients the "Right Way". New York: The Commonwealth Fund, 2011.

10. Bodenheimer T. Coordinating care--a perilous journey through the health care system. N Engl J Med 2008;358:1064-71.

11. Bodenheimer T, Berry-Millett R. Follow the money--controlling expenditures by improving care for patients needing costly services. N Engl J Med 2009;361:1521-3.

12. Minkman MMN. The current state of integrated care: an overview. J Integr Care 2012;20:346-58.

13. Reiss-Brennan B, Briot PC, Savitz LA, et al. Cost and quality impact of Intermountain's mental health integration program. J Healthc Manag 2010;55:97-113. discussion -4.

14. Anderson GF, Ballreich J, Bleich S, et al. Attributes common to programs that successfully treat high-need, high-cost individuals. Am J Manag Care 2015;21:E597-E600.

15. Jaén CR, Crabtree BF, Palmer RF, et al. Methods for evaluating practice change toward a patient-centered medical home. Ann Fam Med 2010;8 Suppl 1(Suppl_1):S9-S20.

16. Reid RJ, Coleman K, Johnson EA, et al. The Group Health Medical Home at year two: cost savings, higher patient satisfaction, and less burnout for providers. Health Aff 2010;29:835-43.

17. Alderwick H, Ham C, Buck D. Population health systems: going beyond integrated care. London: The King's Fund, 2015.

18. Bramwell D, Checkland K, Allen P, et al. Moving Services out of hospital: joining up General Practice and community services? London: Policy Research Unit in Commissioning and the Healthcare System, 2015.

19. Connor M, Kissen G. Tackling whole-systems Change: the Trafford Framework for Integrated Services. J Integr Care 2010;18:4-14.

20. Curry N, Ham C. Clinical and service integration: the route to improved outcomes. London: The King's Fund, 2010.

21. Fillingham D, Weir B. System leadership: lessons and learning from AQUA's Integrated Care Discovery Communities. London: The King's Fund, 2014.

22. Bardsley M, Steventon A, Smith J, et al. Evaluating integrated and community-based care:how do we know what works? London: Nuffield Trust, 2013. 
23. Integrated Health Services - What and why? Geneva: World Health Organization, 2008.

24. Chan AW, Tetzlaff JM, Gøtzsche PC, et al. SPIRIT 2013 explanation and elaboration: guidance for protocols of clinical trials. BMJ 2013;346:e7586.

25. Standards for general practices 4 th edition. East Melbourne: The Royal Australian College of General Practitioners, 2015.

26. Applications of the ACG System in the UK White Paper. The Johns Hopkins University, 2014.

27. Singh D, Ham C. Improving care for people with Long-Term Conditions: a review of UK and International Frameworks. Birmingham: University of Birmingham, 2006.

28. Connor M, Day GE, Meston D. Successful linking of patient records between hospital services and general practice to facilitate integrated care in a hospital and health service in south-east Queensland. Aust Health Rev 2016;40:78-81.

29. Hawthorne G, Korn S, Richardson J. Population norms for the AQoL derived from the 2007 Australian National Survey of Mental Health and Wellbeing. Aust N Z J Public Health 2013;37:7-16.

30. Coast J, Flynn TN, Natarajan L, et al. Valuing the ICECAP capability index for older people. Soc Sci Med 2008;67:874-82.

31. Lubben J, Blozik E, Gillmann G, et al. Performance of an abbreviated version of the Lubben Social Network Scale among three european community-dwelling older adult populations. Gerontologist 2006;46:503-13.

32. Glasgow RE, Wagner EH, Schaefer J, et al. Development and Validation of the patient Assessment of chronic illness care (PACIC). Med Care 2005;43:436-44.

33. Hawthorne G, Sansoni J, Hayes L, et al. Measuring patient satisfaction with health care treatment using the Short Assessment of Patient satisfaction measure delivered Superior and Robust satisfaction estimates. J Clin Epidemiol 2014;67:527-37.

34. Hawthorne G, Richardson J, Osborne R. The Assessment of Quality of Life (AQoL) instrument: a psychometric measure of health-related quality of life. Qual Life Res 1999;8:209-24.
35. Couzner L, Ratcliffe J, Lester L, et al. Measuring and valuing quality of life for public health research: application of the ICECAP-O capability index in the Australian general population. Int $J$ Public Health 2013;58:367-76.

36. Makai P, Brouwer WB, Koopmanschap MA, et al. Quality of life instruments for economic evaluations in health and social care for older people: a systematic review. Soc Sci Med 2014;102:83-93.

37. Glasgow RE, Wagner EH, Schaefer J, et al. Development and validation of the patient Assessment of chronic illness care (PACIC). Med Care 2005;43:436-44.

38. Australian Public Hospitals Cost Report 2013-2014 Round 18. Independent Hospital Pricing Authority. 2016.

39. Vijayaraghavan V, Beasley G. Group Health Cooperative: a case study series on disruptive innovations within integrated health systems. A Health Care Case Study. Innosight Institute, Inc, 2011.

40. Braun V, Clarke V. Using thematic analysis in psychology. Qual Res Psychol 2006;3:77-101.

41. Davies A, Theo C, Bardsley M, et al. Evaluation of complex health andcare interventions using retrospective matched control methods. Report. UK: Nuffield Trust. 6 August 2015.

42. Greaves F, Pappas Y, Bardsley M, et al. Evaluation of complex integrated care programmes: the approach in North West London. Int J Integr Care 2013;13:e006.

43. Steventon A, Bardsley M, Billings J, et al. The role of matched controls in building an evidence base for hospital-avoidance schemes: a retrospective evaluation. Health Serv Res 2012;47:1679-98.

44. Mitchell GK, Burridge L, Zhang J, et al. Systematic review of integrated models of health care delivered at the primary-secondary interface: how effective is it and what determines effectiveness? Aust $J$ Prim Health 2015;21:391-408.

45. Cretin S, Shortell SM, Keeler EB. An evaluation of collaborative interventions to improve chronic illness care. framework and study design. Eval Rev 2004;28:28-51. 